\title{
Role of Computed Tomography in Blunt Abdominal Trauma
}

\author{
Khadka DB ${ }^{1}$, Sharma $A^{1}$, Bhatta $A^{1}$, Maharjan $\mathrm{P}^{1}$, Sharma $\mathrm{S}^{2}$
}

\begin{abstract}
Introduction: Blunt abdominal trauma is one of the commonly encountered surgical emergencies. The diagnostic modality that helps in optimum management of these patients includes chest and abdominal x-rays, Focused Assessment Sonography for Trauma scan and Computed Tomography. In selected hemodynamically stable patients who are candidates for non-operative management, Contrast Enhanced Computed Tomography is not considered essential and hence avoiding its own radiation hazards and decreasing extra financial burden to the patients. Aims: To evaluate whether Contrast Enhanced Computed Tomography is necessary or not in case of blunt trauma abdomen. Methods: This is a hospital based prospective study done in the department of surgery at Nepalgunj Medical College, Kohalpur conducted from October 2020 to March 2021. The patients with blunt abdominal trauma who were hemodynamically stable at the time of presentation and those who became stable after resuscitation were included. These patient's detailed history was taken, clinical examination done. Focused Assessment Sonography for Trauma scan was done at the time of presentation along with chest $\mathrm{x}$-ray and other necessary blood investigation. Data were analyzed with Statistical Package for Social Sciences version 25 and p-value <0.05 was taken as significant. Results: Out of total 53 patients, age group between 11-20 and 21-30 years comprising of 13 patients with male: female ratio of 1.94:1 were affected more. Fall injury, being the most common mode, comprised 20 patients. The commonest organ involved was spleen seen in 17 patients ( $32.1 \%$ ), liver in 16 patients (30.2\%). In $44(83.0 \%)$ patients, Computed Tomography scan was done only in nine patients who were also managed conservatively, except one who underwent laparoscopic evacuation of collected blood. Seventeen (32.1\%) patients underwent repeat ultrasonography without any new findings. Conclusion: Patients with blunt abdominal trauma with stable hemodynamics can be managed conservatively with limited use of Contrast Enhanced Computed Tomography scan.
\end{abstract}

Keywords: Blunt trauma abdomen, Contrast enhanced computed tomography, Fast assessment sonography for trauma

\section{Authors:}

1. Dr. Digbijay Bikram Khadka

2. Prof. Anup Sharma

3. Dr. Ashish Bhatta

4. Dr. PrabirMaharjan

5. Dr. Sandesh Sharma

${ }^{1}$ Department of Surgery, Nepalgunj Medical College and Teaching Hospital, Kohalpur, Banke

${ }^{2}$ Department of Anesthesia,Nepalgunj Medical College and Teaching Hospital, Kohalpur, Banke

\section{Address for Correspondence:}

Dr. Digbijay Bikram Khadka

Lecturer

Department of Surgery

Nepalgunj Medical College and Teaching Hospital

Kohalpur, Banke

Email : iamdigvj@gmail.com

\section{INTRODUCTION}

Blunt trauma abdomen is the injury to intra-abdominal organ which is caused by accidents, falls, assaults or impact without any penetration to visceral organ. ${ }^{1,2,3}$ The most common cause of blunt trauma abdomen in patients include road traffic accident which comprises $75-80 \%$ of all cases. ${ }^{4}$ Liver and spleen are the most commonly injured intra-abdominal organs during blunt abdominal injury., ${ }^{5,6}$ Following liver and spleen, injuries are commonly encountered in kidneys, small bowel, large bowel, pancreas and urinary bladder. ${ }^{7}$ Majority of these injuries can be managed conservatively. The wide spread availability of ultrasound, blood products, ICU set up and laboratory results have shifted the trend to conservative management. Nowadays, focused assessment with sonography for trauma (FAST) is considered the first imaging study in abdominal trauma for those patients who are hemodynamically unstable. ${ }^{8}$ It is done primarily to detect any free fluid in peritoneal cavity, which usually represents hemoperitoneum. If present, further imaging and/or surgery may be required. ${ }^{9}$ The advantages of CT scan are its ability to grade the solid organ injury as well as identification of ongoing bleeding in the form of contrast extravasation if present. However, CT scan is criticized to be an expensive investigation which has the potential to miss hollow 
viscus injuries. ${ }^{10,11}$ The drawbacks of radiation injury and cost effectiveness along with unavailability in primary center makes it troublesome. ${ }^{12,13}$ In our teaching hospital, we frequently encounter patients with blunt trauma abdomen but study has not been done for non-operative/conservative management of patients without the need for CT scan.

\section{METHODS}

The prospective study was carried out in the Department of Surgery, Nepalgunj Medical College and Teaching Hospital, Kohalpur from October 2020 to March 2021. Ethical clearance was obtained from the institutional review committee, Nepalgunj Medical College and Teaching Hospital before conducting the study. This study included 53 patients who presented with blunt abdominal trauma who were hemodynamically stable or who became stable after initial resuscitation. However, patients with ongoing intraperitoneal bleed, peritonitis or passage of fresh blood in digital rectal examination and pts who became unstable while being treated conservatively and were excluded. Demographic data at the time of admission was taken. A detailed history was taken. A thorough clinical examination was performed. Following laboratory investigations were obtained from each patient at the time of admission: complete blood count, renal function test, serum amylase, random blood sugar, serology, chest x-ray (erect), ultrasound abdomen and pelvis. The patients who were hemodynamically stable at presentation and throughout the hospital stay, did not undergo CECT abdomen, however repeat USG abdomen and pelvis was done when necessary. Patients were closely monitored in surgical ward with serial hemoglobin and abdominal girth measurement. Patients who became unstable in the due course of conservative management were excluded. The end point was the successful conservative management of selective patients without abdominal CT scan. Data analysis was performed with SPSS 25.

\section{RESULTS}

The total number of patients included in this study was 53. Throughout the study period, 70 patients presented to Nepalgunj Medical College and Teaching Hospital with abdominal trauma. Among them, 17 patients were excluded from the study; out of which, seven patients hemodynamic status worsened while being on non-operative management, six patients had gas under diaphragm and underwent exploratory laparotomy and three patients had head injury and one patient had pelvic injury. Majority of the patients were of 11-20 and 21-30 years of age while least were age group 51-60. The most common mode of injury leading to abdominal trauma was fall injury comprising of $20(37.7 \%)$ cases followed by physical assault and road traffic accidents. Seventeen (32.1\%) patients with blunt abdominal trauma had splenic injury, making it a commonly affected organ. Liver was second most common comprising $16(30.2 \%)$ patients. There were $8(15.2 \%)$ patients who had multiple organ involvement (Figure 1). About 18.9\% had gross hemoperitoneum, 35.8\% had mild hemoperitoneum and $45.3 \%$ had moderate hemoperitoneum (Table II). At presentation, 49 patients with blunt abdominal trauma were stable. The hemodynamic stability was categorized on the basis of systolic and diastolic blood pressure, pulse and hemoglobin at presentation. Four patients with unstable hemodynamics also responded to fluid resuscitation and thus, were included in this group.

\begin{tabular}{|lcc|}
\hline \multicolumn{1}{|c}{ CT Abdomen } & Frequency & Percent \\
\hline Done & 9 & 17 \\
Not Done & 44 & 83 \\
Total & $\mathbf{5 3}$ & 100 \\
\hline
\end{tabular}

Table I: CT Abdomen

\begin{tabular}{lcc}
\multicolumn{1}{l}{$\begin{array}{c}\text { USG (FAST) Findings } \\
\text { Hemoperitoneum }\end{array}$} & Frequency & Percentage \\
Mild & 19 & 35.8 \\
Moderate & 24 & 45.3 \\
Gross & 10 & 18.9 \\
Total & $\mathbf{5 3}$ & $\mathbf{1 0 0 . 0}$
\end{tabular}

Table II: USG (FAST) Findings

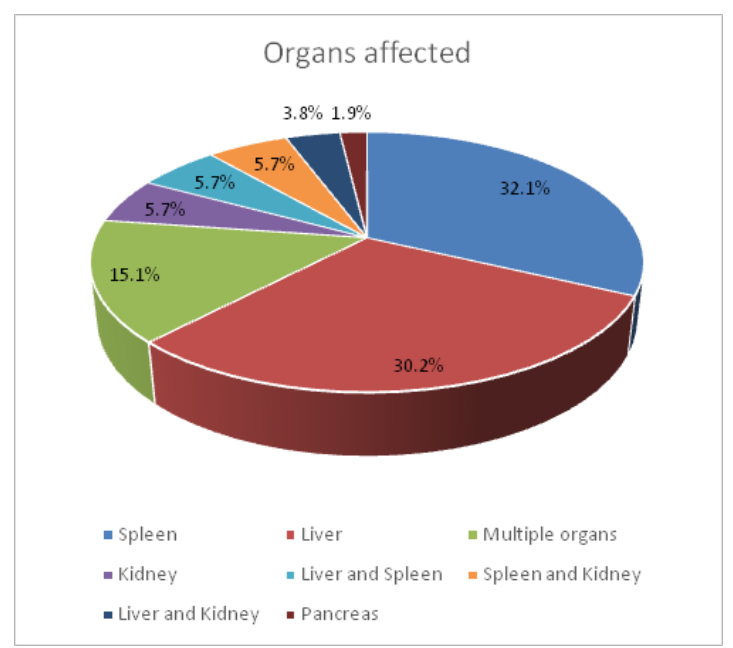

Figure I: Organs affected

Of the total patients, CT abdomen was done in only 9(17\%) patients during the course of treatment (Table I). The CT abdomen in these was done due to clinical deterioration like increasing distension of abdomen or persisting pain and not because of change in hemodynamics (hypotension). Among them, 3 each had isolated liver and splenic I injury and remaining 3 had multivisceral injury. All these were also managed conservatively. When the total expenditure was compared between those who had undergone CT scan during the course of treatment (9 patients) with rest; it was found that the expenditure was slightly higher in those who underwent CT abdomen. 


\section{DISCUSSION}

The total number of patients were 53. In our study, majority of the patients were of $11-20$ and $21-30$ years of age accounting for $24.5 \%$ in each age group. Mehta $\mathrm{N}$ et al (2014) studied 72 patients with BTA and revealed that the predominant age group was $21-30$ years accounting for $40 \%$ of the patients. ${ }^{15}$ Out of the 53 patients included in our study $66 \%$ were males while $36 \%$ were females. Study done by Mehta N et al. in 2014 also revealed majority of the patients with blunt trauma abdomen were males accounting for $79 \%$ of total patients and $21 \%$ were females. ${ }^{15}$ The male to female ratio in their study was 3.7:1 whereas in our study the ratio was 1.94:1. It has been reported that trauma usually affects the young productive aged group that eventually poses economic burden to the nation as well as on families. ${ }^{16}$

Our study revealed that amongst the abdominal organs involved, spleen was most commonly injured $(n=17,32.1 \%)$ followed by liver $(n=16,30.2 \%)$. Pancreas was the least commonly affected organ among 53 patients $(n=1,1.9 \%)$. Particularly, among multiple trauma patients, abdomen is the third most frequently injured body part. ${ }^{17}$ A study done by Mehta $\mathrm{N}$ et al. also showed similar findings where spleen was the most commonly injured organ accounting for 53\% of the total patients under the study. ${ }^{15}$ Similarly, Davis J et al conducted a study where they found out that, spleen (62\%) was the most common organ involved followed by liver $(51 \%) .{ }^{18}$ The least commonly affected solid organ was pancreas accounting for 8 patients only. Similar observation was noted by Morton $J$ et al where organs affected in decreasing order were spleen $(37 \%)$, liver (5\%) and pancreas (2\%). ${ }^{19}$ Compared from various studies were similar to our studies. However there are several studies which reported liver to be the most common injured solid organ followed by spleen in blunt abdominal trauma. ${ }^{20,21}$

In our study, the mode of injury revealed a slightly different pattern as compared to different studies. Our observation was that $37.7 \%$ patients presented with BTA due to fall injury followed by physical assault (32.1\%) and road traffic accidents (30.2\%). In a study done by Mehta $\mathrm{N}$ et al. motor vehicle accidents $(53 \%)$ were the most common mode of injury. ${ }^{15}$ Similarly, in a study done by Davis J et al. 65 also incriminated automobile accidents (70\%) as the most common mode. In their study, blow to the abdomen (17\%) was the second most common presentation and fall injuries being the least, approximately 6\%. Another study done by Morton J et al. revealed automobile accidents (33\%) followed by fall injuries $(17 \%)$ and blow to abdomen (13\%) as the common modes of injuries. ${ }^{19}$ The differences in the observations could be attributed to the geographical location of our country and livelihood of people. There are few studies whose findings are similar to ours. ${ }^{22,23}$ These studies were done in Saudi Arabia and was explained by the construction boom leading to increased morbidity and mortality among workers falling from height at a construction site.

In our study, we preferred conservative management of patients who were initially hemodynamically stable at presentation and those who became stable after initial resuscitation without the CT abdomen. Of the 53 patients, only one patient had to undergo diagnostic laparoscopy and drainage of the collected blood and the rest were discharged after varying days of hospital stay. Various studies in the pediatric age group have supported the non-operative management specifically in splenic and hepatic injuries. Traumotologists have started to adopt the conservative management approach for management of patients with BTA without evidence of hollow viscus injury/peritonitis. ${ }^{14}$ In our study, USG initial at presentation was able to identify hemoperitoneum (mild $35.8 \%$, moderate $-45.3 \%$, gross $-18.9 \%$ ), solid organ injuries (splenic/hepatic injuries) and intra-abdominal collections. The role of USG as an initial diagnostic tool in management of BTA has also been emphasized by Sunkara SB et al. ${ }^{24}$ Their study concluded that USG is a non-invasive, fast, portable bedside modality without radiation hazards with advantage that it can be repeated when on doubt without any contraindication. Non-operative management was successful and only USG was done as a radiological modality of investigation. Similar study was made by Kumar S et al. in 2017, where 84 patients were enrolled in the study. ${ }^{12}$ Patients were taken up for selective non operative management (SNOM) with or without CECT abdomen. Forty-four patients were included in their SNOM group. SNOM without CECT was successful in 36 (81.82 \%) which was similar to our study. Their study concluded that SNOM can be practiced safely in patients of abdominal trauma with limited use of CECT scan. In our study, it was observed that patients who underwent CT had to bear extra cost for the investigation and suffered more financial burden compared to the patients who did not undergo CT scan with similar outcome.

Poletti et $\mathrm{al}^{25}$ suggested that in order to reduce medical costs and unnecessary radiation exposure evidence based guidelines need to be implemented. They conducted a study where they described that FAST, chest radiography and 3 laboratory parameters (increased serum glutamic oxaloacetic transaminase, increased white blood count, and hematocrit of $36 \%$ ), a normal abdominal examination and GCS 13 could safely exclude abdominal injuries without using CT.

CECT is an advanced investigation and the facility and expertise may not always be available, especially in rural areas and even in urban parts in underdeveloped countries. Furthermore, it adds to the cost to the patient, the risk of contrast allergy and nephropathy. For CT scan patient needs to be shifted to the CT scan where clinicians loses active observation on a critically injured patients, especially with airway intubation and chest drains. Feasibility of repeat CT if needed is also very poor because of its expensiveness. FAST or even a detailed USG of abdomen and pelvis especially in stable patients is cheap, less time consuming, free of risk of contrast adverse effects and easily available. It can be repeated multiple times also.

\section{LIMITATIONS}

The major limitation is that this is a descriptive study, with limited sample size. The results can be made more accurate and statistically significant by conducting a randomized controlled 
trial to disprove the common belief that CT abdomen is a must in abdominal trauma. For this we need to have a longer time period and larger sample size.

\section{CONCLUSION}

Blunt abdominal trauma is common in younger age group and males are commonly affected. Fall injury is the common mode of injury in our result. Spleen is the commonest solid organ to be involved. Blunt abdominal trauma in patient with stable hemodynamics or in those who responds to initial resuscitation can be managed without CT scan. Careful and detailed evaluation with clinical examination and USG abdomen can give the same outcome. Majority of patients without hollow viscous perforation or without peritonitis can be managed successfully without surgery.

\section{REFRENCES}

1. Shojaee $M$, Dolatabadi AA, Sabzghabaei A, et al. New scoring system for intra-abdominal injury diagnosis after blunt trauma. Chin J Traumatol. 2014;17(1):19-24.

2. Calder BW, Vogel AM, Zhang J, et al. Focused assessment with sonography for trauma in children after blunt abdominal trauma: a multi-institutional analysis. J Trauma Acute Care Surg. 2017;83(2):218-24.

3. Huntimer CM, Muret-Wagstaff S, Leland NL. Can falls on stairs result in small intestine perforations?. J Pediatr. 2000;106(2):301-5.

4. Schwab CW. Selection of nonoperative management candidates. World J surg. 2001;25(11):1389-92.

5. Becker CD, Mentha G, Terrier F. Blunt abdominal trauma in adults: role ofCT in the diagnosis and management of visceral injuries Part 1: liver and spleen. Eur. Radiol. 1998;8(4):55362.

6. Kozar RA, Crandall M, Shanmuganathan K, et al. Organ injury scaling 2018update:spleen, liver, and kidney. J TraumaAcute Care Surg. 2018;85(6):1119-22.

7. Kong JP, Bultitude MF, Royce P, Gruen RL, Cato A, Corcoran NM. Lower urinary tract injuries following blunt trauma: a review of contemporary management. Rev Urol. 2011;13(3):119.

8. Jansen JO, Yule SR, Loudon MA. Investigation of blunt abdominal trauma.BMJ. 2008;336(7650):938-42.

9. Smith J. Focused assessment with sonography in trauma (FAST): should its role be reconsidered?. Postgrad Med J. 2010;86(1015):285-91.

10. Marx JA, Moore EE, Jorden RC, Eule Jr JO. Limitations of computed tomography in the evaluation of acute abdominal trauma: a prospective comparison with diagnostic peritoneal lavage. J Trauma. 1985;25(10):933-7.

11. Meyer DM, Thal ER, Weigelt JA, Redman HC. Evaluation of computed tomography and diagnostic peritoneal lavage in blunt abdominal trauma.J Trauma Acute Care Surg.
1989;29(8):1168-72.

12. Kumar S, Prakash P, Joshi MK, Rathi V. Selective non-operative management ofpatients with abdominal trauma-is CECT scan mandatory?. Indian J Surg. 2017;79(5):396-400.

13. Dehqanzada ZA, Meisinger Q, Doucet J, Smith A, Casola G, Coimbra R.Complete ultrasonography of trauma in screening blunt abdominal trauma patients is equivalent to computed tomographic scanning while reducing radiation exposure and cost. J Trauma Acute Care Surg. 2015;79(2):199-205.

14. StawickiSP. Trends in nonoperative management of traumatic injuries-A synopsis. Int J CritIIInInj Sci. 2017;7(1):38.

15. Mehta N, Babu S, Venugopal K. An experience with blunt abdominal trauma: evaluation, management and outcome. ClinPract. 2014;4(2):599.

16. Salimi J, Ghodsi M, Zavvarh MN, Khaji A. Hospital management of abdominal trauma in Tehran, Iran: A review of 228 patients. Chin. J. Traumatol. 2009;12(5):259-62.

17. Abdelrahman H, Ajaj A, Atique S, El-Menyar A, Al-Thani $\mathrm{H}$. Conservative management of major liver necrosis after angioembolization in a patient with blunt trauma. Case Rep Surg. 2013;2013:954050. doi: 10.1155/2013/954050.

18. Davis JJ, Cohn Jr IS, Nance FC. Diagnosis and management of blunt abdominal trauma. . Ann Surg. 1976;183(6):672.

19. Morton JH, Hinshaw JR, Morton JJ. Blunt trauma to the abdomen. . Ann Surg. 1957;145(5):699.

20. Smith J, Caldwell E, D'Amours S, Jalaludin B, Sugrue M. Abdominal trauma: a disease in evolution. ANZ J Surg. 2005;75(9):790-4.

21. Clancy TV, Maxwell JG, Covington DL, Brinker CC, Blackman D. A statewide analysis of level I and II trauma centers for patients with major injuries. J Trauma Acute Care Surg.. 2001;51(2):346-51.

22. Karamercan A, Yilmaz TU, Karamercan MA, Aytaç B. Blunt abdominal trauma: evaluation of diagnostic options and surgical outcomes. Ulus TravmaAcilCerrahiDerg. 2008;14(3):205.

23. Gad MA, Saber A, Farrag $S$, Shams ME, Ellabban GM. Incidence, patterns, and factors predicting mortality of abdominal injuries in trauma patients. NAm J Med Sci. 2012;4(3):129.

24. Sai Bharat Sunkara, Biranchi Narayan Lenka, Gaurav Jha, TreenaMinz, SaikKasifSahajada. Role of ultrasound in management of blunt abdominal trauma. J. Evid. Based Med. Health. 2019;6(29):1927-33.

25. Poletti PA, Mirvis SE, Shanmuganathan K, et al. Blunt abdominal trauma patients: can organ injury be excluded without performing computed tomography?. J Trauma. 2004;57(5):1072-81. 